\title{
The Timing of Emotional Discrimination in Human Amygdala and Ventral Visual Cortex
}

\author{
Dean Sabatinelli, ${ }^{1}$ Peter J. Lang, ${ }^{2}$ Margaret M. Bradley, ${ }^{2}$ Vincent D. Costa, ${ }^{2}$ and Andreas Keil ${ }^{2}$ \\ ${ }^{1}$ Department of Psychology and BioImaging Research Center, University of Georgia, Athens, Georgia 30602, and ${ }^{2}$ Center for the Study of Emotion and \\ Attention, University of Florida, Gainesville, Florida 32611
}

\begin{abstract}
Models of visual emotional perception suggest a reentrant organization of the ventral visual system with the amygdala. Using focused functional magnetic resonance imaging in humans with a sampling rate of $100 \mathrm{~ms}$, here we determine the relative timing of emotional discrimination in amygdala and ventral visual cortical structures during emotional perception. Results show that amygdala and inferotemporal visual cortex differentiate emotional from nonemotional scenes $\sim 1 \mathrm{~s}$ before extrastriate occipital cortex, whereas primary occipital cortex shows consistent activity across all scenes. This pattern of discrimination is consistent with a reentrant organization of emotional perception in visual processing, in which transaction between rostral ventral visual cortex and amygdala originates the identification of emotional relevance.
\end{abstract}

\section{Introduction}

Visual perception of emotionally arousing, relative to nonemotional, stimuli is associated with greater blood oxygen leveldependent (BOLD) signal across widespread regions of the ventral visual system, including inferotemporal (IT) and extrastriate occipital cortex (Bradley et al., 2003; Norris et al., 2004; Sabatinelli et al., 2005, 2007; Britton et al., 2006). Although considerable evidence suggests a relationship between activity in amygdala and inferotemporal cortex during emotional perception (Morris et al., 1998; Armony and Dolan, 2002; Vuilleumier et al., 2004; Sabatinelli et al., 2005), the means by which ventral visual system differentiates emotional from nonemotional scenes is not well defined.

Visual emotional discrimination in nonhuman primates is hypothesized to result from reentrant feedback from the amygdala to ventral visual cortex (Spiegler and Mishkin, 1981; Amaral and Price, 1984; Iwai and Yukie, 1987). Specifically, Amaral and colleagues identify dense amygdala innervation into rostral inferotemporal cortex, with more sparse innervation in caudal occipital areas (Amaral et al., 1992, 2003; Freese and Amaral, 2005). This dense interconnectivity, the high correlation between amygdala and inferotemporal activity (Morris et al., 1998; Armony and Dolan, 2002; Vuilleumier et al., 2004; Sabatinelli et al., 2005), and the role of the inferotemporal cortex in high-level visual perception (DeYoe and Van Essen, 1988; Chao et al., 1999; Grill-Spector and Malach, 2004) suggests that the process of emotional discrimination in human visual perception may originate during the interaction between amygdala and rostral inferotemporal cortex and develops later in caudal extrastriate cortex. If emotional dis-

\footnotetext{
Received July 9, 2009; revised Sept. 11, 2009; accepted 0ct. 13, 2009.

This work was supported by National Institute of Mental Health Grant P50-MH-072850.

Correspondence should be addressed to Dean Sabatinelli, 523 Psychology Building, University of Georgia,

Athens, GA 30602.E-mail: sabat@uga.edu.

DOI:10.1523/JNEUROSCI.3278-09.2009

Copyright $\odot 2009$ Society for Neuroscience $\quad 0270-6474 / 09 / 2914864-05 \$ 15.00 / 0$
}

crimination in amygdala and inferotemporal cortex precedes emotional discrimination in occipital cortex, a reentrant model of emotional perception would be supported. If no difference in the timing of emotional discrimination is evident across extrastriate occipital cortex, IT cortex, and amygdala, the reentrant model may be insufficiently described, the recording methodology used here too insensitive, or alternative models relevant to emotional perception exclusive of amygdala feedback may be more appropriate (Heller, 1990; Posner and Petersen, 1990).

It is possible to discriminate cortical sources in ventral visual cortex with surface-based electroencephalography (Sabatinelli et al., 2007; Keil et al., 2009), yet spatial resolution is considerably reduced in rostral inferior temporal areas, in which the distance from the scalp is greatest (Russell et al., 1998; Schoffelen and Gross, 2009). Conversely, differentiating the timing of activation in visual cortex is beyond the temporal resolution of whole-brain functional magnetic resonance imaging (fMRI) acquisition techniques. However, although BOLD contrast is inherently delayed relative to neural activity, the timing of signal change within active clusters is highly reliable (Kim et al., 1997; Menon and Kim 1999; Miezin et al., 2000). By comparing the time course of BOLD signal within regions of interest (ROI) across experimental conditions, the effective temporal resolution is limited only by the sampling rate at which BOLD signal can be recorded and the reliability of the signal. Because we are not concerned with the relative timing of BOLD activation across regions, potential confounds regarding the variations in vascular anatomy, as well as individual differences in BOLD timing (Aguirre et al., 1998; Buxton et al., 1998), are avoided.

Here we record BOLD signal in amygdala and inferotemporal, extrastriate, and striate occipital cortices in a single slice, as rapidly as signal quality will allow, while participants view an eventrelated series of emotionally arousing and neutral pictures. If emotional discrimination in visual perception originates via feedback between amygdala and rostral inferotemporal cortex, emotional discrimination in these regions (differential BOLD 
Table 1. Normative ratings of the IAPS picture stimuli used in the current study

\begin{tabular}{lll}
\hline & Valence & Arousal \\
\hline Pleasant & $6.71(0.36)$ & $6.27(0.32)$ \\
Neutral & $5.27(0.58)$ & $3.34(0.27)$ \\
Unpleasant & $1.68(0.18)$ & $6.75(0.42)$ \\
\hline
\end{tabular}

Valence and arousal are rated on a scale of $1-9$, with SD in parentheses.

signal across arousing and neutral picture conditions) should occur earlier than in caudal occipital cortex.

\section{Materials and Methods}

Participants. Twenty undergraduate volunteers participated for course credit or $\$ 20$ compensation. All volunteers consented to participate after reading a description of the study, approved by the local human subjects review board. Before entering the bore of the Siemens $3 \mathrm{~T}$ Allegra MR scanner, participants were fitted with earplugs and given a patient-alarm squeeze ball. A vacuum pillow, padding, and explicit verbal instruction were used to limit head motion. Two participants' data were excluded because of excessive head motion, and one was lost as a result of scanner malfunction. The final sample included 10 males and 7 females (average age, 18.8 years; range, $18-21$ years).

Stimuli and procedure. Participants were asked to maintain fixation on a dot at the center of a 7 inch liquid crystal display screen mounted directly behind the head, visible via a coil-mounted mirror (IFIS MRcompatible hardware; Intermagnetics). After three acclimation trials in which checkerboard stimuli were presented, a series of 24 picture stimuli were presented $\left(25^{\circ}\right.$ visual angle $)$ in an event-related design. The picture stimuli were chosen from the International Affective Picture System (IAPS) (Lang et al., 2008) (http://csea.phhp.ufl.edu/Media.html) and all depicted people, including eight exemplars each of highly arousing erotic couples (pleasant: 4611, 4658, 4659, 4669, 4676, 4680, 4690, and 4694), moderately arousing neutral people (neutral: 2037, 2102, 2305, 2383, $2393,2396,2513$, and 2595), and highly arousing mutilations (unpleasant: 3000, 3030, 3060, 3068, 3069, 3100, 3102, and 3225). The pleasant and unpleasant pictures were selected to be equivalent in normative ratings of emotional arousal (Table 1). All picture stimuli were converted to grayscale and matched for luminance and $90 \%$ quality JPEG file size by category using Adobe Photoshop 7 (Adobe Systems). Each picture was presented for $3 \mathrm{~s}$, followed by a $9 \mathrm{~s}$ fixation-only period. Picture order was pseudorandomized, allowing no more than two successive presentations of a stimulus category. The picture series was repeated (in unique orders) in three additional blocks, for a total of 96 trials over $\sim 23 \mathrm{~min}$.

Scanning parameters. Once participants were comfortable inside the bore, an 8 min T1-weighted three-dimensional structural volume was collected. The prescription specified 160 sagittal slices, with $1 \mathrm{~mm}$ isotropic voxels in a $256 \mathrm{~mm}$ field of view. In addition, a single T1-weighted slice was acquired at the location of the single functional slice acquisition, described below. After structural acquisitions, the $5 \mathrm{~mm}$ slice prescription (gradient echo; echoplanar $64 \times 64 ; 180 \mathrm{~mm}$ field of view; $25^{\circ}$ flip angle; $30 \mathrm{~ms}$ echo time; $100 \mathrm{~ms}$ repetition time) was oriented in an oblique axial plane such that sampling of amygdala, inferotemporal cortex, and middle occipital gyrus could be obtained. The placement was tailored for each participant, originating with coverage of amygdala, and tilted for optimum coverage of visual areas of interest, and if possible, to exclude sinus cavity coverage, as a means of limiting susceptibility artifact. This led to substantial sampling of the calcarine fissure. The singleslice prescription allows $40 \mathrm{ml}$ voxels to be repetitively sampled at a temporal resolution of $100 \mathrm{~ms}$.

Data reduction. Each participant's 96-trial functional time series was linearly detrended, temporally smoothed with a $1 \mathrm{~s}$ Gaussian filter, and spatially smoothed across 2 voxels $(5.625 \mathrm{~mm}$ full-width at halfmaximum) using BrainVoyager QX 1.8 (Brain Innovation). Temporal smoothing was necessary to reduce the effects of physiological noise present in the time series at a $10 \mathrm{~Hz}$ sampling rate. Trials with residual head motion were removed manually, by identifying large (greater then four times the background variation) and brief spikes located by examining the average time series intensity across a majority of the voxels in

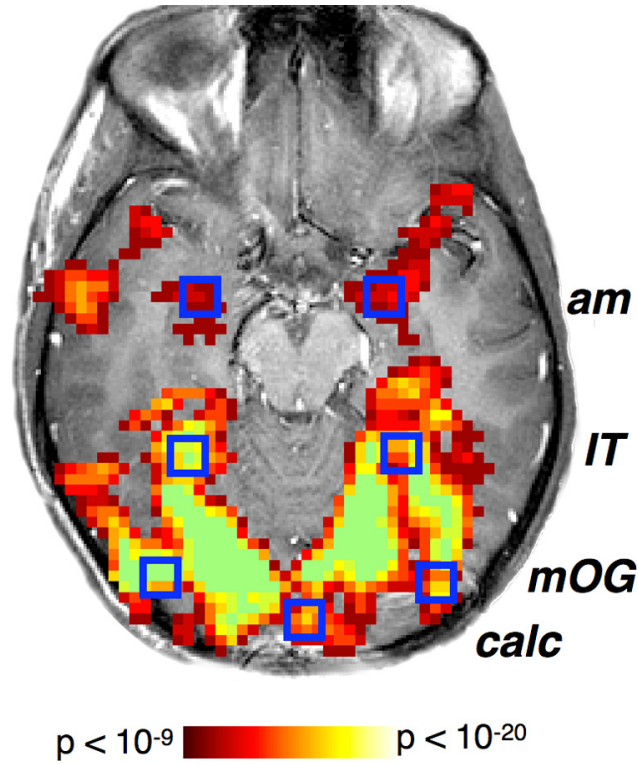

Figure 1. Example of statistical overlay of BOLD signal for a single subject. Regions of interest (blue boxes) were derived from significant activity resulting from picture presentation in bilateral amygdala (am), IT, middle occipital gyrus (m0G), and calcarine fissure (calc).

the slice (a rectangular region of greater than half the voxels within the brain). This procedure resulted in the removal of $<2 \%$ of total trials and no more than four trials from any subject. Average signal-tonoise [( signal - noise)/SD noise] ratios calculated from the image data were 80.5 in calcarine fissure, 81.0 in middle occipital gyrus, 97.8 in inferotemporal cortex, and 79.4 in amygdala.

The processed image series were entered into single-subject ANOVAs, identifying BOLD signal change evoked by the three picture contents (erotica, neutral people, and mutilations), using a standard two-gamma hemodynamic response function (Boynton et al., 1996). A false discovery rate (Genovese et al., 2002) of $p<0.01$ was used to threshold each participant's data. From these functional maps, four regions were sampled, including bilateral amygdala, inferotemporal cortex, middle occipital gyrus, and midline calcarine fissure. Each region was sampled across 9 voxels $(356 \mathrm{ml})$ using axial neuroanatomical atlases (Talairach and Tournoux, 1988; Haines, 1995) as guides (Fig. 1). The variability of placement across subjects for all ROIs was minimized as much as possible, balancing the need for consistency across subjects with sensitivity to the central location of significant clusters within subject.

\section{Results}

The effect of picture content on BOLD signal at the peak of the response (4-8 s after picture onset) was greater during pleasant and unpleasant, relative to neutral, picture presentations in middle occipital gyrus (content $F_{(2,15)}=49.93, p<0.001$; quadratic $\left.F_{(1,16)}=64.34, p<0.001\right)$, inferotemporal cortex (content $F_{(2,15)}=26.67, p<0.001$; quadratic $F_{(1,16)}=51.62, p<$ 0.001 ), and amygdala (content $F_{(2,15)}=6.02, p<0.05$; quadratic $\left.F_{(1,16)}=12.72, p<0.01\right)$. Pleasant and unpleasant pictures led to equivalent BOLD signal increase across the three regions (no linear effects approached significance). No effects of picture content were found in calcarine fissure.

To reliably identify the point at which emotion-specific BOLD signal increases occurred in amygdala and inferotemporal and middle occipital gyri, nonparametric permutation tests (Maris, 2004; Maris and Oostenveld, 2007) were computed for each time point and region in the first $5 \mathrm{~s}$ (50 time points) of picture presentation. Labels encoding picture arousal (pleasant and unpleasant pictures led to equivalent responses) were randomly reassigned in 10,000 draws and checked for independence from 

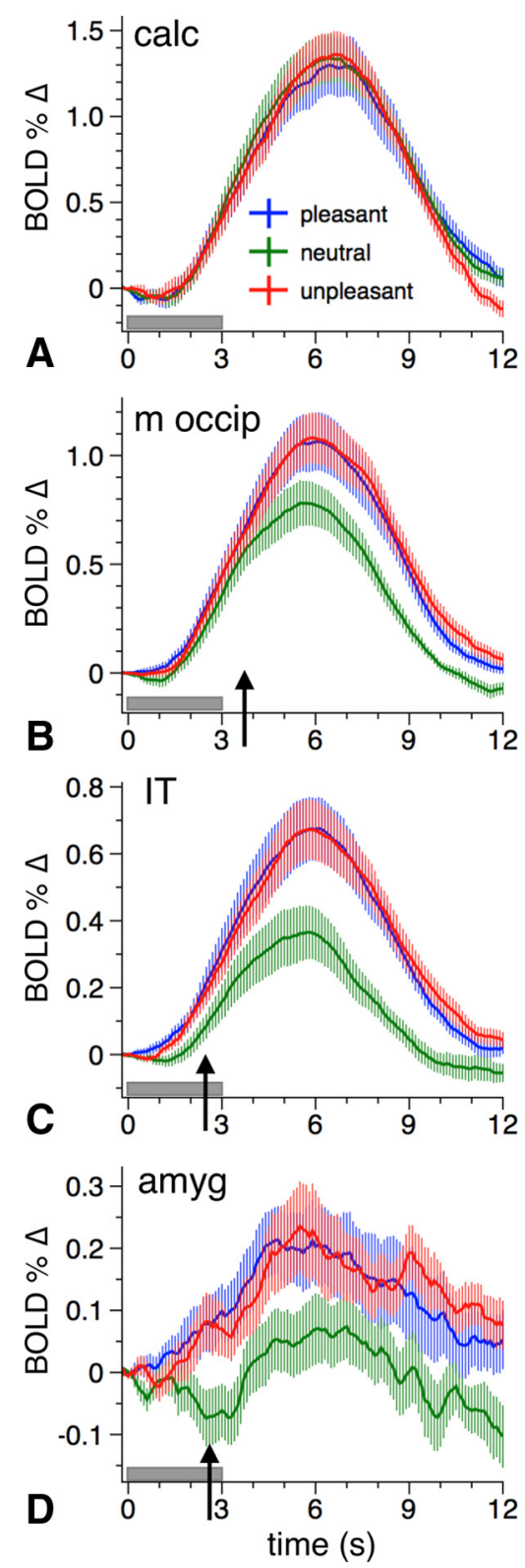

Figure 2. Average time courses $(n=17)$ of picture-driven BOLD signal in amygdala, inferotemporal cortex, middle occipital gyrus, and calcarine fissure. Blue and red lines represent pleasant and unpleasant pictures, and green represents neutral pictures. The gray bar on the abscissa signifies the picture presentation period. Arrows indicate the point at which BOLD signal during an arousing picture presentation is significantly greater activity than BOLD signal during neutral pictures.

previous permutation orders. A repeated measures $F$ statistic was then generated for each time point, and a Gaussian function was fit to the distribution. The value of the $F$ statistic used in forming the permutation distribution was computed as the 99.9th percentile of the distribution described by this fitted Gaussian $(p<$ 0.01 ). The time after picture onset at which this threshold was met are $3.9 \mathrm{~s}$ for middle occipital gyrus, $2.5 \mathrm{~s}$ for inferotemporal cortex, and $2.9 \mathrm{~s}$ for amygdala (Fig. 2, arrows on the abscissa).

A second test including structure (inferotemporal and middle occipital ROIs) and emotional discrimination (arousing and nonarousing pictures) as factors yielded an interaction of ROI and arousal that was reliable $(p<0.05)$ from 3.4 to $3.9 \mathrm{~s}$ after picture onset, indicating a significantly earlier discrimination of emotional arousal in inferotemporal regions relative to middle occipital gyrus.

\section{Discussion}

These data show that emotion-related increases in BOLD signal change are reliable in amygdala and inferotemporal visual cortex $\sim 1$ s before extrastriate occipital cortex. Because the sequence of basic visual processing stages places extrastriate cortex (V2) ahead of inferotemporal areas (DeYoe and Van Essen, 1988; Desimone and Ungerleider, 1989), evidence of later discrimination supports the perspective (Amaral et al., 1992; Lang et al., 1997; Shi and Davis, 2001; Vuilleumier, 2005) that emotional significance is identified by some means in the amygdala, through which feedback to rostral IT, and eventually caudal occipital areas, leads to enhanced perceptual processing and "motivated attention."

The integration of emotional significance into visual perception as a result of amygdala - rostrocaudal recurrent processing fits well with conceptions of complex scene processing as an iterative, non-hierarchical mechanism (Lamme and Roelfsema, 2000; Grill-Spector and Malach, 2004; Hegdé and Felleman, 2007). In the primate, the initial inferotemporal cortical response to a picture of a conspecific is thought to reflect global categorization of the percept and is followed by a more sustained response that it associated with detail factors such as identity and facial expression (Nakamura et al., 1994; Sugase et al., 1999; Nishijo et al., 2008). The timing of this later stage of detail processing is consistent with estimates of categorization latency in human research (Junghöfer et al., 2001; VanRullen and Thorpe, 2001; Codispoti et al., 2006; Tsuchiya et al., 2008). Two intracranial studies have shown human amygdala differentiation of aversive from neutral pictures (Oya et al., 2002) and facial expressions (Krolak-Salmon et al., 2004) beginning 150-200 ms after stimulus onset. We speculate that, in the current dataset, it is this later processing stage that may underlie the increased signal present in amygdala and IT cortex, necessarily delayed and smoothed through hemodynamic BOLD contrast.

The statistical thresholds resulting from the permutation resampling procedure enabled us to identify the time point at which BOLD signal in our regions of interest emotionally arousing trials differed from nonarousing trials. This analysis shows that the amygdala and inferotemporal cortex discriminated picture emotionality before middle occipital gyrus. However, the inferotemporal cortex shows statistically reliable differentiation before amygdala (2.5 vs $2.9 \mathrm{~s}$ after picture onset) (Fig. 2). However, a two-factor test of amygdala and inferotemporal arousal discrimination yields no interaction, and thus the difference in discrimination onset is not reliable. Perhaps more importantly, a demonstration that the inferotemporal cortex does not differentiate emotional stimuli in the absence of amygdala input (Vuilleumier et al., 2004) suggests that the discrimination originates in the amygdala and is brought about in inferotemporal areas soon thereafter.

Other means to assess the timing of BOLD signal can approximate high temporal resolution of neural activity, such as image acquisition jitter and modeling of undersampled responses (Lee et al., 2006; Duff et al., 2007; Fuhrmann Alpert et al., 2007). In a study of fear-relevant picture processing (Larson et al., 2006), amygdala activity was recorded in spider phobics and controls using a jittered image acquisition, with an effective sampling rate of $300 \mathrm{~ms}$. Modeling of the BOLD signal response yielded a reduced latency of $>1 \mathrm{~s}$ in phobics relative to controls in response to spider pictures yet no difference in signal amplitude. Modeling 
of BOLD signal is undoubtedly a powerful means of assessing the time course of BOLD signal yet is dependent on the accurate specification of many underlying factors. Here we intended to exploit the capability of data collection to the fullest extent and thus reduce the chance of mischaracterization of the data.

Although the timing of emotional discrimination in amygdala and inferotemporal cortex relative to occipital cortex is consistent with a reentrant model of emotional perception, the support is inferential. Beyond the simple timing of emotion differentiation across structures, predictive time series techniques such as Granger causality analyses could provide support for the superordinate role of amygdala differentiation in emotional perception. In the framework of Granger causality, one measured process is said to be causal to a second if the predictability of the second process at a given time point is improved by including measures from the history of the first process. The current dataset did not lend itself to such analyses, because the brief picture periods and eventrelated design precluded stationary neural processing states of sufficient duration. Future work using fast-sampled fMRI and experimental designs allowing extended, stable periods of emotional processing may be more suitable.

The early difference (1-3 s) in amygdala signal across picture contents can be attributed to both an early increase during arousing pictures and a transient decrease at the onset of neutral pictures. This decrease in amygdala signal in response to nonarousing conditions in studies of emotional processing has been reported in several fMRI studies (Wright et al., 2001; Armony and Dolan, 2002; Morris et al., 2002), but the mechanism is as yet unknown. Future work explicitly controlling the predictability of stimulus conditions and timing may shed light on this possibility.

A tradeoff of focused image acquisition is reduced coverage. This study is intended to test the hypothesis that specific regions of the visual system differed from another in the timing of BOLD change across conditions, which were capable of being sampled in a single plane with the amygdala. Of course there may be other areas of the brain that show emotional differentiation at earlier or later points, and these possibilities can be addressed in additional studies.

In summary, these data show in a human sample the relative timing of emotional discrimination across amygdala and ventral visual cortical structures during emotional perception. In this analysis, amygdala and inferotemporal cortex differentiate emotional from nonemotional scenes $\sim 1$ s before secondary occipital cortex, whereas primary occipital cortex shows consistent activity across all scenes. This pattern of discrimination is consistent with a reentrant organization of emotional perception in visual processing, in which transaction between rostral ventral visual cortex and amygdala originates the identification of emotional relevance.

\section{References}

Aguirre GK, Zarahn E, D'Esposito M (1998) The variability of human BOLD hemodynamic responses. Neuroimage 8:360-369.

Amaral DG, Price JL (1984) Amygdalo-cortical projections in the monkey (Macaca fascicularis). J Comp Neurol 230:465-496.

Amaral DG, Price JL, Pitkanen A, Carmichael ST (1992) Anatomical organization of the primate amygdaloid complex. In: The amygdala: neurobiological aspects of emotion, memory, and mental dysfunction (Aggleton JP, ed), pp 1-66. New York: Wiley.

Amaral DG, Behniea H, Kelly JL (2003) Topographical organization of projections from the amygdala to the visual cortex of in the macaque monkey. Neuroscience 118:1099-1120.

Armony JL, Dolan RJ (2002) Modulation of spatial attention by fear- conditioned stimuli: an event-related fMRI study. Neuropsychologia 40:817-826.

Britton JC, Taylor SF, Sudheimer KD, Liberzon I (2006) Facial expression and complex IAPS pictures: common and differential networks. Neuroimage 31:906-919.

Boynton GM, Engel SA, Glover GH, Heeger DJ (1996) Linear systems analysis of functional magnetic resonance imaging in human V1. J Neurosci 16:4207-4221.

Bradley MM, Sabatinelli D, Lang PJ, Fitzsimmons JR, King W, Desai P (2003) Activation of the visual cortex in motivated attention. Behav Neurosci 117:369-380.

Buxton RB, Wong EC, Frank LR (1998) Dynamics of blood flow and oxygenation changes during brain activation: the balloon model. Magn Reson Med 39:855-864.

Chao LL, Martin A, Haxby JV (1999) Are face-responsive regions selective only for faces? Neuroreport 10:2945-2950.

Codispoti M, Ferrari V, De Cesarei A, Cardinale R (2006) Implicit and explicit categorization of natural scenes. Prog Brain Res 156:53-65.

Desimone R, Ungerleider LG (1989) Neural mechanisms of visual processing in monkeys. In: Handbook of neuropsychology (Boller F, Grafman J, eds), pp 267-299. Amsterdam: Elsevier.

DeYoe EA, Van Essen DC (1988) Concurrent processing streams in monkey visual cortex. Trends Neurosci 11:219-226.

Duff E, Xiong J, Wang B, Cunnington R, Fox P, Egan G (2007) Complex spatio-temporal dynamics of fMRI BOLD: a study of motor learning. Neuroimage 34:156-168.

Freese JL, Amaral DG (2005) The organization of projections from the amygdala to visual cortical areas TE and V1 in the macaque monkey. J Comp Neurol 486:295-317.

Fuhrmann Alpert G, Sun FT, Handwerker D, D’Esposito M, Knight RT (2007) Spatio-temporal information analysis of event-related BOLD responses. Neuroimage 34:1545-1561.

Genovese CR, Lazar NA, Nichols T (2002) Thresholding of statistical maps in functional neuroimaging using the false discovery rate. Neuroimage 15:870-878.

Grill-Spector K, Malach R (2004) The human visual cortex. Ann Rev Neurosci 27:649-677.

Haines DE (1995) Neuroanatomy: an atlas of structures, sections, and systems, Ed 4. Baltimore: Williams and Williams.

Hegdé J, Felleman DJ (2007) Reappraising the functional implications of the primate visual anatomical hierarchy. Neuroscientist 13:416-421.

Heller W (1990) The neuropsychology of emotion: developmental patterns and implications for psychopathology. In: Psychological and biological approaches to emotion. (Stein NL, Leventhal B, Trabasso T, eds), pp 167-211. Hillsdale, NJ: Erlbaum.

Iwai E, Yukie M (1987) Amygdalofugal and amygdalopetal connections with modality-specific visual cortical areas in macaques (Macaca fuscata, M. mulatta, and M. fascuicularis). J Comp Neurol 261:362-387.

Junghöfer M, Bradley MM, Elbert TR, Lang PJ (2001) Fleeting images: a new look at early emotion discrimination. Psychophysiology 38:175-178.

Keil A, Sabatinelli D, Ding M, Lang PJ, Ihssen N, Heim S (2009) Re-entrant projections modulate visual cortex in affective perception: evidence from Granger causality analysis. Hum Brain Mapp 30:532-540.

Kim SG, Richter W, Uğurbil K (1997) Limitations of temporal resolution in functional MRI. Magn Reson Med 37:631-636.

Krolak-Salmon P, Hénaff MA, Vighetto A, Bertrand O, Mauguière F (2004) Early amygdala reaction to fear spreading in occipital, temporal, and frontal cortex: a depth electrode ERP study in human. Neuron 42:665-676.

Lamme VA, Roelfsema PR (2000) The distinct modes of vision offered by feedforward and recurrent processing. Trends Neurosci 23:571-579.

Lang PJ, Bradley MM, Cuthbert BN (1997) Motivated attention: affect activation and action. In: Attention and orienting: sensory and motivational processes (Lang PL, Simons RF, Balaban MT, eds), pp 97-135. Hillsdale NJ: Erlbaum.

Lang PJ, Bradley MM, Cuthbert BN (2008) International affective picture system (IAPS): affective ratings of pictures and instruction manual. Technical Report A-8. Gainesville, FL: University of Florida.

Larson CL, Schaefer HS, Siegle GJ, Jackson CA, Anderle MJ, Davidson RJ (2006) Fear is fast in phobic individuals: amygdala activation in response to fear-relevant stimuli. Biol Psychiatry 60:410-417.

Lee L, Friston K, Horwitz B (2006) Large-scale neural models and dynamic causal modelling. Neuroimage 30:1243-1254. 
Maris E (2004) Randomization tests for ERP topographies and whole spatiotemporal data matrices. Psychophysiology 41:142-151.

Maris E, Oostenveld R (2007) Non-parametric tests of EEG and MEG data. J Neurosci Methods 164:177-190.

Menon RS, Kim SG (1999) Spatial and temporal limits in cognitive neuroimaging with fMRI. Trends Cogn Sci 3:207-216.

Miezin FM, Maccotta L, Ollinger JM, Petersen SE, Buckner RL (2000) Characterizing the hemodynamic response: effects of presentation rate, sampling procedure, and the possibility of ordering brain activity based on relative timing. Neuroimage 11:735-759.

Morris JS, Friston KJ, Büchel C, Frith CD, Young AW, Calder AJ, Dolan RJ (1998) A neuromodulatory role for the human amygdala in processing emotional expressions. Brain 121:47-57.

Morris JS, deBonis M, Dolan RJ (2002) Human amygdala responses to fearful eyes. Neuroimage 17:214-222.

Nakamura K, Matsumoto K, Mikami A, Kubota K (1994) Visual properties of single neurons in the temporal pole of behaving monkeys. J Neurophysiol 71:1206-1221.

Nishijo H, Hori E, Tazumi T, Ono T (2008) Neural correlates to both emotion and cognitive functions in the monkey amygdala. Behav Brain Res 188:14-23.

Norris CJ, Chen EE, Zhu DC, Small SL, Cacioppo JT (2004) The interaction of social and emotional processes in the brain. J Cogn Neurosci 16:18181829.

Oya H, Kawasaki H, Howard MA 3rd, Adolphs R (2002) Electrophysiological responses in the human amygdala discriminate emotion categories of complex visual stimuli. J Neurosci 22:9502-9512.

Posner MI, Petersen SE (1990) The attention system of the human brain. Annu Rev Neurosci 13:25-42.

Russell GS, Srinivasan R, Tucker DM (1998) Bayesian estimates of error bounds for EEG source imaging. IEEE Trans Med Imaging 17:1084-1089.

Sabatinelli D, Bradley MM, Fitzsimmons JR, Lang PJ (2005) Parallel amyg- dala and inferotemporal activation reflect emotional intensity and fear relevance. Neuroimage 24:1265-1270.

Sabatinelli D, Bradley MM, Lang PJ, Costa VD, Versace F (2007) Pleasure rather than salience activates human nucleus accumbens and medial prefrontal cortex. J Neurophysiol 98:1374-1379.

Schoffelen JM, Gross J (2009) Source connectivity analysis with MEG and EEG. Hum Brain Mapp 30:1857-1865.

Shi C, Davis M (2001) Visual pathways involved in fear conditioning measured with fear-potentiated startle: behavioral and anatomic studies. J Neurosci 21:9844-9855.

Spiegler BJ, Mishkin M (1981) Evidence for the sequential participation of inferior temporal cortex and amygdala in the acquisition of stimulusreward associations. Behav Brain Res 3:303-317.

Sugase Y, Yamane S, Ueno S, Kawano K (1999) Global and fine information coded by single neurons in the temporal visual cortex. Nature 400: $869-873$.

Talairach J, Tournoux P (1988) Co-planar stereotaxic atlas of the human brain. New York: Thieme.

Tsuchiya N, Kawasaki H, Oya H, Howard MA 3rd, Adolphs R (2008) Decoding face imformation in time, frequency and space from direct intracranial recordings of the human brain. PLOS One 3:e3892.

VanRullen R, Thorpe SJ (2001) The time course of visual processing: From early perception to decision-making. J Cogn Neurosci 13:454-461.

Vuilleumier P (2005) How brains beware: neural mechanisms of emotional attention. Trends Cogn Sci 12:585-594.

Vuilleumier P, Richardson MP, Armony JL, Driver J, Dolan RJ (2004) Distant influences of amygdala lesion on visual cortical activation during emotional face processing. Nat Neurosci 7:1271-1278.

Wright CI, Fischer H, Whalen PJ, McInerney SC, Shin LM, Rauch SL (2001) Differential prefrontal cortex and amygdala habituation to repeatedly presented emotional stimuli. Neuroreport 12:379-383. 